Review Article

\title{
Improving Cognitive Function from Children to Old Age: A Systematic Review of Recent Smart Ageing Intervention Studies
}

\author{
Rui Nouchi ${ }^{1,2}$ and Ryuta Kawashima ${ }^{2,3}$ \\ ${ }^{1}$ Human and Social Response Research Division, International Research Institute of Disaster Science, Tohoku University, \\ 4-1 Seiryo-cho, Aoba-ku, Sendai 980-8575, Japan \\ ${ }^{2}$ Department of Advanced Brain Science, Smart Ageing International Research Center, Institute of Development, \\ Aging and Cancer, Tohoku University, 4-1 Seiryo-cho, Aoba-ku, Sendai 980-8575, Japan \\ ${ }^{3}$ Department of Functional Brain Imaging, Institute of Development, Aging and Cancer, Tohoku University, \\ 4-1 Seiryo-cho, Aoba-ku, Sendai 980-8575, Japan
}

Correspondence should be addressed to Rui Nouchi; rnouchi@idac.tohoku.ac.jp

Received 12 March 2014; Accepted 30 June 2014; Published 11 August 2014

Academic Editor: Daniela Schulz

Copyright (C) 2014 R. Nouchi and R. Kawashima. This is an open access article distributed under the Creative Commons Attribution License, which permits unrestricted use, distribution, and reproduction in any medium, provided the original work is properly cited.

\begin{abstract}
Background. Cognitive functions are important for daily life at any age. One purpose of Smart Ageing is to investigate how to improve cognitive functions. This systematic review evaluates beneficial effects of the intervention on cognitive functions. Method. We conducted a systematic review of intervention studies of improvements of cognitive functions published or in press before December 2013. Because of the heterogeneity of the intervention programs, a systematic and critical review of the interventions and outcomes was conducted instead of a meta-analysis. Results. We identified nine completed and published studies, which were divided into four categories: cognitive training using video game, cognitive training using PC, cognitive training using paper and pencil, and exercise training. Review results showed that various intervention programs can improve cognitive functions such as executive functions, working memory, episodic memory, processing speed, and general cognitive ability/IQ. Conclusions. The systematic review demonstrated that some intervention programs can be effective for improving various aspects of cognitive functioning at any age. Some limitations to this review include its small sample size and heterogeneity of programs and cognitive function measures, in addition to unresolved issues such as transfer of everyday skills and effectiveness for nonhealthy people.
\end{abstract}

\section{Background}

Cognitive function includes a variety of mental processes such as perception, attention, memory, decision making, and language comprehension. Cognitive function serves a critical role in everyday behavior and social behavior. For instance, when one goes shopping, it is necessary to memorize information about what to buy, how to make a proper judgment to buy, and how to have a conversation with shop assistants. Considering communication with a friend, we identify the friend by looking at faces or hearing a voice and sharing information with the friend.

Our cognitive functions change during our lifetimes [14]. Cognitive functions improve from childhood to young adulthood. Some cognitive functions such as executive functions and working memory reach a peak during 20 s or 30s [5]. However, semantic knowledge (semantic memory) develops to the age of 60 or 70 [6]. An elderly person might experience a decline of several cognitive functions, including memory [3, 7], attention [8], executive functions $[9,10]$, and processing speed [11]. Previous studies have demonstrated that higher cognitive functions in children and young adults are positively correlated with higher academic achievements [12-17]. A decline in the cognitive abilities of older people has been shown to engender difficulty in performing basic activities of daily living [18-23]. Consequently, improvements of cognitive functions using intervention programs are attracting attention at all age levels.

Confirming expectations of society and scientific fields related to improvement of cognitive functions, we have undertaken Smart Ageing research (http://www2.idac.tohoku.ac.jp/dep/sairc/index.html). The Smart Ageing concept 
includes a positive acceptance of later stages in life and a perspective of aging as a series of "developmental stages toward intellectual maturity." Smart Ageing is a revolutionary paradigm shift away from negative concepts, such as antiaging, which imply an unwillingness to accept or face later stages of life. One purpose of Smart Ageing research is to investigate how to develop the cognitive functions of healthy children, how to retain them in healthy adults, and how to improve them in community-dwelling seniors because cognitive functions serve a critical role in everyday life. To achieve that purpose, we have conducted widely varied intervention studies. Ours is a rare case of one laboratory that has conducted intervention studies to assess cognitive functions in subjects from children to older people.

Previous review papers demonstrated that some intervention programs can improve cognitive functions in healthy and nonhealthy children, young adults, and older adults [24-38]. Intervention programs were divided into interventions of two main types: cognitive training and exercise training. Cognitive training was defined as an intervention that provides structured practice on tasks related to aspects of cognitive functioning such as memory, attention, language, and executive function. There are many types of cognitive training such as working memory training $[28,39,40]$, processing speed training [38, 41], memory strategic training [24, 42-44], and brain training game [45-47]. Some cognitive training intervention studies using randomized controlled trials (RCT) have shown improvements of cognitive functions [44, 45, 4751]. There are two types of exercise training such as aerobic exercise training and strength exercise training [36]. Aerobic exercise training is defined as structured exercise programs involving the use of large muscle groups for extended periods of time in activities that are rhythmic in nature, including but not limited to walking, stepping, running, swimming, cycling, and rowing [32]. Strength exercise training uses resistance against the force of muscular contraction to build strength, anaerobic endurance, and skeletal muscle mass. Strength exercise training often uses gravity to oppose muscle contraction [34, 52, 53]. Earlier studies using RCT have revealed that aerobic exercise training alone and strength exercise training alone improved cognitive functions $[32,53$, 54].

Given that the research area of intervention for improvements of cognitive function is growing rapidly, it is important to review evidence of recent intervention studies and to ascertain the methodological quality of intervention studies. For intervention studies, transfer effects especially are important phenomena. A transfer effect is defined as "the ability to extend what has been learned in one context to new contexts" [55]. Additionally, the transfer effect is classifiable in terms of a near transfer effect and a far transfer effect [56-59]. The near transfer effect refers to improvements in cognitive domains that are related closely to the trained cognitive processes. In contrast, the far transfer effect refers to improvements in cognitive domain that are not closely related to the trained cognitive processes. It is necessary to evaluate whether intervention programs had near or far transfer effects. Consequently, in the systematic review, we evaluate the beneficial effects of the intervention on cognitive functions and the methodological quality of recent intervention studies for Smart Ageing.

\section{Methods}

2.1. Search Strategy. In accordance with the preferred reporting items for systematic reviews and meta-analysis (PRISMA) statement [60], we conducted a systematic review of intervention studies of improvements of cognitive functions for Smart Ageing published or in press before December 2013. To identify relevant studies, we searched PubMed, Google Scholar, and PsycINFO using the following key words for cognitive function: "cognitive," "cognitive abilities," "cognition," "memory", and "speed of processing"; for interventions: "treatment," "training," "intervention," "randomized controlled trials", or "RCT"; for author: "kawashima" and "ryuta." We searched references of included papers and relevant systematic reviews. We also asked experts in the field if they knew of other related papers meeting inclusion criteria. Supporting PRISMA checklist is available as Supplemental Materials (see Supplementary Material available online at http://dx.doi.org/10.1155/2014/235479).

2.2. Inclusion and Exclusion Criteria. We included studies in healthy participants who had no diagnosis such as developmental disorder, depression, posttraumatic stress disorder (PTSD), dementia, mild cognitive impairment, or Alzheimer's disease. Studies must have been published in English and must have used standardized psychological tests or tools to measure cognitive functions. We excluded single case reports, dissertations, meeting abstracts, study protocols, and review papers. We also excluded studies which measured no cognitive functions.

2.3. Quality Assessment. The quality of each study was assessed using a modified Delphi list [61]. To enhance the quality of assessment, additional elements were considered, including details of random allocation methods, adequate description of the control/comparison group, betweengroup statistical comparison, reporting dropouts, and reporting consolidated standards of reporting trials (CONSORT) statement (http://www.consort-statement.org/). These quality assessment criteria were based on a previous systematic review paper. The total score $(\operatorname{Max}=14)$ is reported in Table 1.

2.4. Statistical Analysis. Because of the heterogeneity of the intervention programs, types of participants, outcomes assessed, and measurement tools used, a systematic and critical review of the interventions and outcomes was conducted instead of a meta-analysis.

\section{Results}

The database search strategy initially yielded 25 journal articles (20 articles from PubMed, Google Scholar, and PsycINFO and 5 articles from an expert's suggestions). Based on the stated inclusion and exclusion criteria, 9 of the 25 


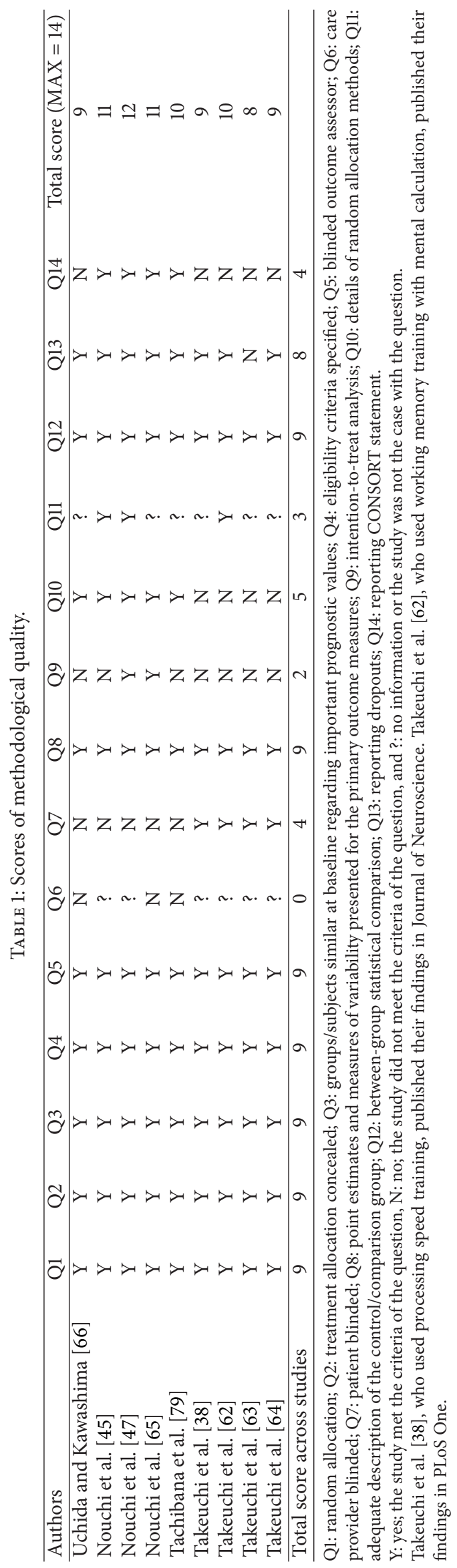




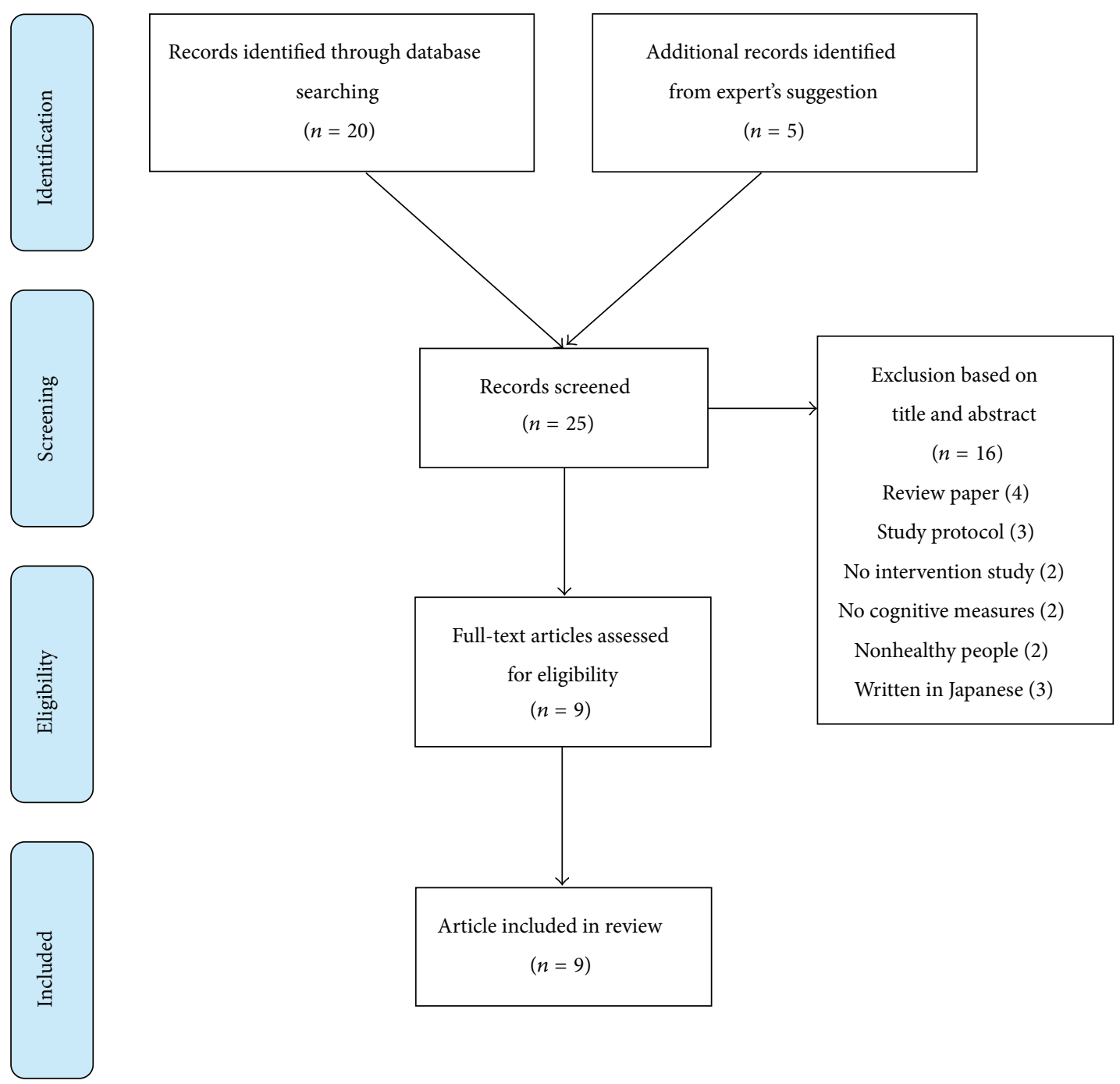

FIgURE 1: Flow diagram of search results.

publications [38, 45, 47, 62-67] were eligible for the current review (Figure 1). We present details of these interventions in short descriptions.

Of the 16 excluded articles, four articles were review articles discussing cognitive intervention [28, 68-70]. Three articles were excluded because they were protocols for intervention [71-73]. Two articles were not intervention studies $[74,75]$. Two articles did not measure any cognitive functions $[76,77]$. Two articles were excluded because the participants had been diagnosed as having dementia or autism spectrum disorder $[78,79]$. Three articles were written in Japanese [8082].

3.1. Quality Assessment. An assessment of the methodological quality of the included studies is presented in Table 1. The quality assessment ranged from 8 to 12 , with an average of 9.89 ( $\mathrm{SD}=1.27)$. All included studies had sufficient good methodological quality. A study by Nouchi et al. [65] had the highest methodological quality. The score of item 6 (care-provider blinded) was low among the included studies because no care providers were involved in six studies that used PC-based training programs or video games [38, 45, 47, 62-64]. The score of item 11 (adequate description of the control/comparison group) was also low because of the use of a passive control or a waiting list control group [38, 63-67]. Four studies [38, 62-64] investigated changes of brain structure or neural mechanisms though cognitive training. Therefore, the studies did not use an intentionto-treat (ITT) analysis. Consequently, the score of item 9 (using ITT analysis) was low. However, all studies met the methodological qualities of item of $1,2,3,4,5,8$, and 12 .

3.2. Participants, Sample Size, and Duration of Intervention. Among the nine studies, one study included preschool children (4-6 years old) [67], five studies included young adults (undergraduate and graduate students) [38, 47, 6264], and three studies included elderly people (over 60 years old) $[45,65,66]$. The numbers of participants in the nine studies were from $32[45,47]$ to 236 [67]. The durations of intervention were from 6 days $[38,62]$ to 23 weeks [66]. 
A six-day intervention was used in two studies [38, 62]. A four-week intervention was used in five studies $[45,47,63-$ $65]$. Two studies used intervention periods of longer than 3 months $[66,67]$.

3.3. Methods of Intervention Programs. Table 2 presents characteristics of the intervention programs. Two studies used video games such as the popular brain training game (Brain Age) and popular puzzle games (Tetris) [45, 47]. Four studies used PC-based cognitive training such as working memory training, processing speed training, and multitask training [38, 62-64]. Two studies used paper-pencil-based intervention programs $[66,67]$. One study used exercise training [65]. Four studies used an active control condition $[45,47,62,66]$, where the other five used a waiting list or notraining control group [38, 63-65, 67].

3.4. Cognitive Function Measures, Analysis Methods, and Significant Findings. To evaluate the beneficial effects of intervention on cognitive functions, a wide variety of cognitive functions measures was used. Most measures were standardized psychological measures. They showed high reliability and validity. Measures of the cognitive functions were divided into seven domains: general cognitive functions/intelligence quotient (IQ), executive functions, episodic memory, working memory/short-term memory, attention, processing speed, and others (Table 3 ).

To measure general cognitive functions/IQ, five studies for young people [38, 47, 63, 64] used Raven's advanced progressive matrices test. Two studies of elderly people [45, 66] used the minimental state examination as a measure of general cognitive function/IQ. To measure executive functions, the Stroop test was widely used [38, 47, 63-65]. To measure working memory/short-term memory, seven studies used digit span $[38,45,47,63-65]$. Three studies used digit cancellation task as a measure of attention [45, 47, 65]. Five studies used symbol coding as a measure of processing speed $[45,47,62,65,66]$.

Seven studies used analysis of covariance (ANCOVA) with a change score (posttest minus pretest scores) to detect group differences [38, 45, 47, 62-65]. Two studies used $t$ tests to investigate the group differences $[66,67]$. Table 4 shows the significant effects after interventions. Despite the methodological heterogeneity of the interventions, eight studies found the beneficial effects of the interventions on the cognitive functions compared to control groups [38, 45, $47,62-65,67]$. One study found no significant improvements of cognitive functions but showed significant improvement of cognitive functions in the target intervention group compared to control groups.

3.5. Short Descriptions of Eligible Articles. Next we present a short description of the intervention study. Based on the intervention programs, the included studies were divided into four categories (cognitive training using video game, cognitive training using PC programs, cognitive training using paper-pencil programs, and exercise training).
3.5.1. Cognitive Training Using Video Game Training Game. Video game training is one type of cognitive training. Two studies $[45,47]$ investigated the beneficial effects of brain training games on the cognitive functions. These studies used the same training periods ( 15 min per day, 5 days per week for 4 weeks) and the same brain training games (Brain Age) as a target intervention. Brain Age is a popular brain training game. Based on earlier neuroscience evidence, Brain Age was developed by Ryuta Kawashima. Most games in Brain Age include elements of these reading aloud and simple arithmetic calculations. For example, in the calculation 20 game, participants must answer 20 simple arithmetic calculations as quickly as possible. The questions include problems of mathematical addition, subtraction, and multiplication. In reading aloud, participants must read aloud excerpts from Japanese classical literature. In syllable count, some sentences written in a combination of kanji and kana are presented. Participants must count the kana letters after translating kanji to kana. These studies used a popular puzzle game (Tetris) as active control groups. In Tetris, players rotate and move blocks descending from the top of the screen so that these blocks form lines at the bottom of the screen. After a complete line with no gaps is formed, the line disappears and points are awarded. If no line is formed, then the blocks pile higher and higher until the block pile reaches the top of the screen, at which point the game ends and the player loses. The goal is to keep the game going as long as possible by forming complete lines with the descending blocks. As the game progresses, the descending blocks give players less time to choose where to place each block. For elderly people [45], the brain training game improved executive functions measured using the frontal assessment battery and trail making test and processing speed measured by symbol coding and symbol search measured processing speed compared to the puzzle game. For young people [47], the brain training game led to improved executive functions measured using the Wisconsin card sorting test and Stroop test, working memory measured by the operation span, letter-number sequence, and arithmetic, and processing speed measured by symbol coding and symbol search compared to the puzzle game. However, the puzzle game improved attention measured by the simple reaction task and visuospatial ability measured by mental rotation.

3.5.2. Cognitive Training Using PC Programs. There were several types of cognitive training using PC. Two studies were working memory training $[62,63]$. One study was processing speed training [38]. One study was multitask training [64]. Participants of all included studies were young people. These studies also measured changes of brain structure and functions after interventions. We specifically examined the improvement of cognitive functions. Therefore, this report describes only results of cognitive functional change. Our other reviews have reported effects of cognitive intervention on neural systems $[28,69]$.

The processing speed training program consisted of adaptive training of processing speed tasks [77]. Training tasks involved eight tasks using computer buttons and two tasks using paper and pencil. In all, eight training tasks using 


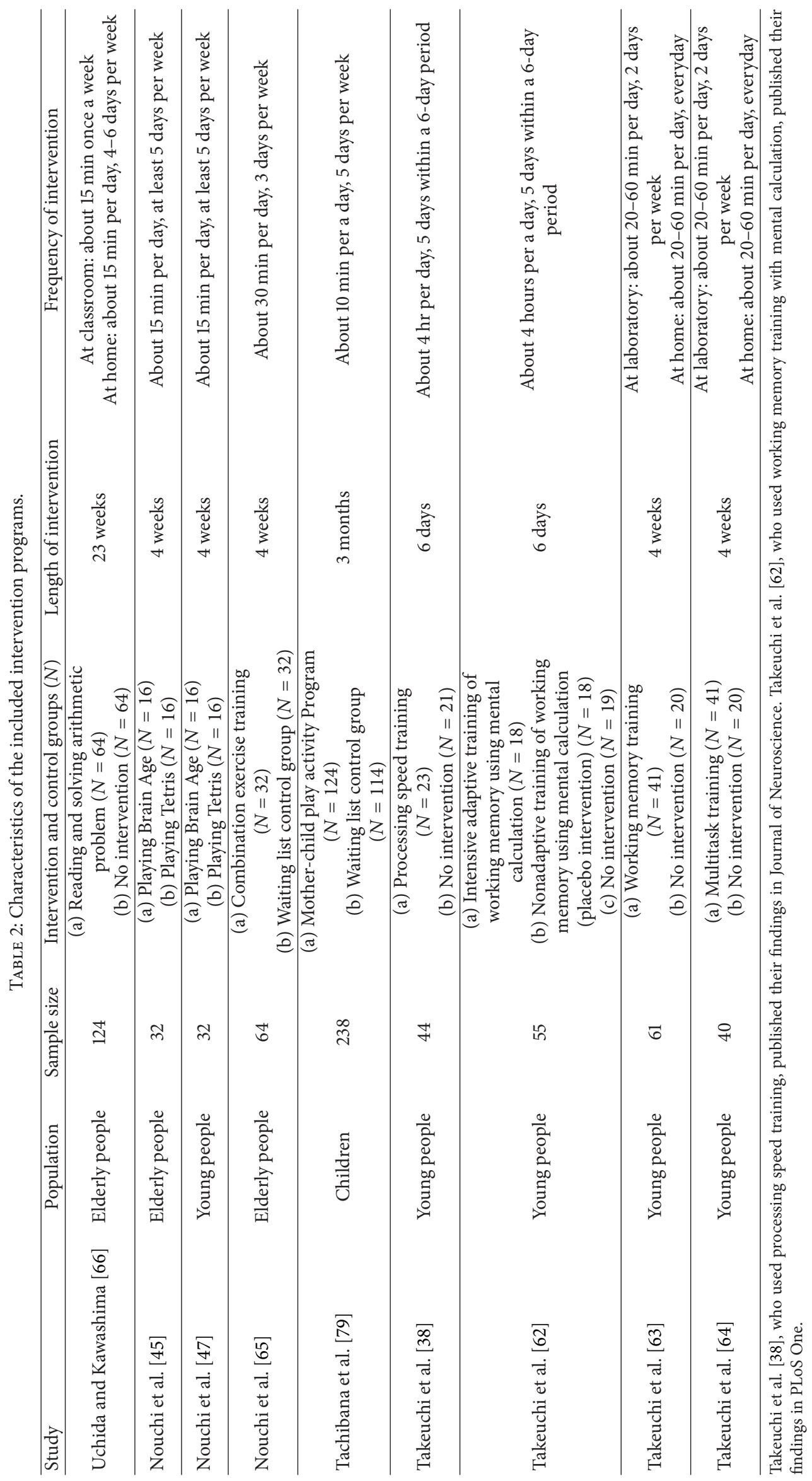




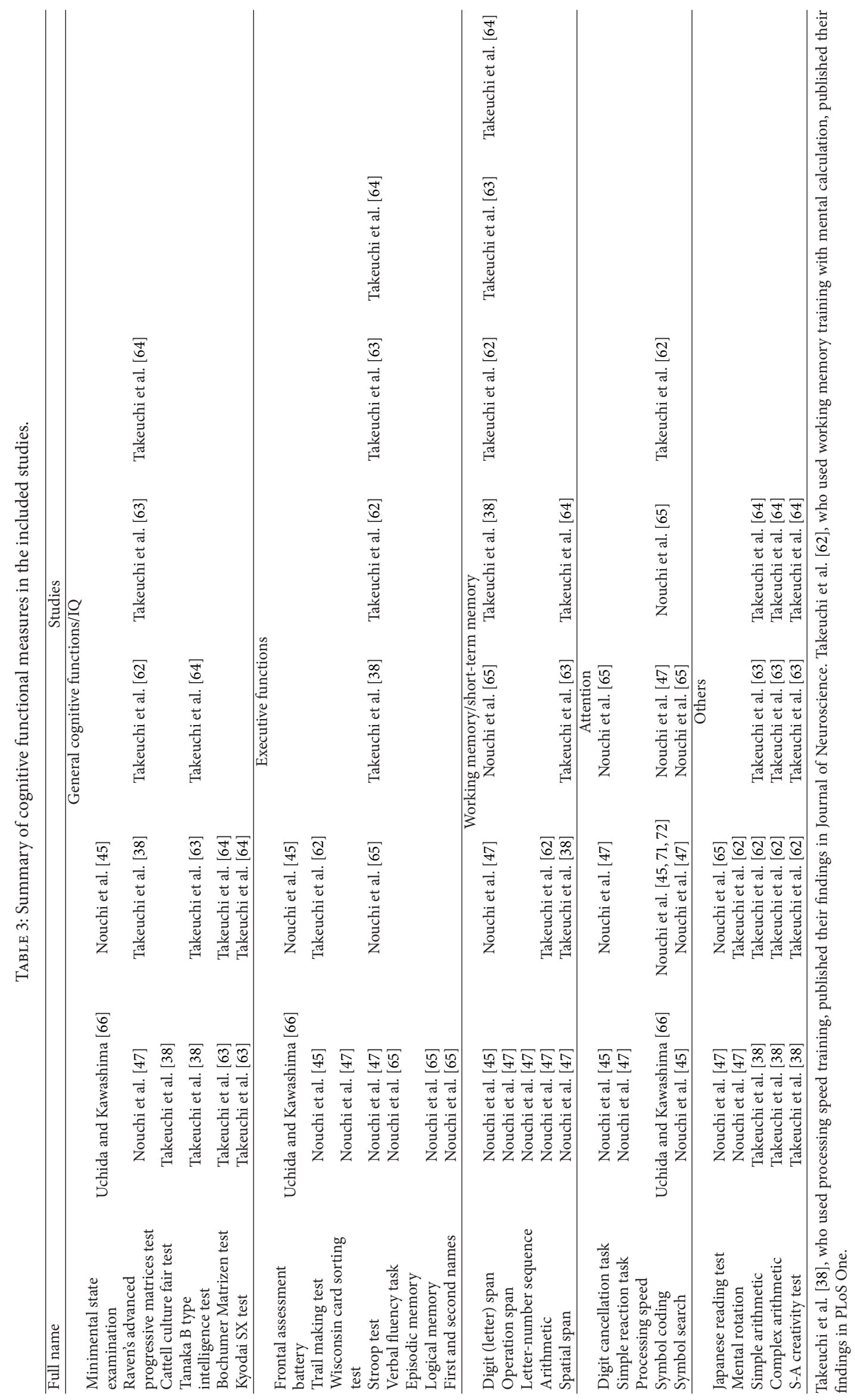


TABLE 4: Summary of improvements of cognitive function after intervention programs.

\begin{tabular}{|c|c|c|}
\hline Study & Measures of cognitive functions & Significant effect of target intervention group \\
\hline \multirow{3}{*}{ Uchida and Kawashima [66] } & MMSE & 0 \\
\hline & FAB & 0 \\
\hline & $\mathrm{Cd}$ & 0 \\
\hline \multirow{8}{*}{ Nouchi et al. [45] } & MMSE & 0 \\
\hline & FAB & + \\
\hline & Trail making test & + \\
\hline & D-CAT & 0 \\
\hline & DS-F & 0 \\
\hline & DS-B & 0 \\
\hline & $\mathrm{Cd}$ & + \\
\hline & SS & + \\
\hline \multirow{17}{*}{ Nouchi et al. [47] } & RAPMT & 0 \\
\hline & WCST & + \\
\hline & rST & + \\
\hline & ST & + \\
\hline & OpS & + \\
\hline & LNS & + \\
\hline & Ari & + \\
\hline & DS-F & 0 \\
\hline & DS-B & 0 \\
\hline & SpS-F & 0 \\
\hline & SpS-B & 0 \\
\hline & D-CAT & 0 \\
\hline & SRT & - \\
\hline & $\mathrm{Cd}$ & + \\
\hline & SS & + \\
\hline & JART & 0 \\
\hline & MR & - \\
\hline \multirow{12}{*}{ Nouchi et al. [65] } & LFT & + \\
\hline & CFT & + \\
\hline & $\mathrm{rST}$ & + \\
\hline & ST & + \\
\hline & LM & + \\
\hline & FSN & 0 \\
\hline & DS-F & 0 \\
\hline & DS-B & 0 \\
\hline & D-CAT & 0 \\
\hline & $\mathrm{Cd}$ & + \\
\hline & SS & + \\
\hline & JART & 0 \\
\hline \multirow{8}{*}{ Tachibana et al. [79] } & DAM & + \\
\hline & Understanding relationships between things & 0 \\
\hline & Counting and comparing the numbers & 0 \\
\hline & Calculation & 0 \\
\hline & Completion of the pictures & 0 \\
\hline & Working memory & + \\
\hline & Processing speed & + \\
\hline & Total score & 0 \\
\hline
\end{tabular}


TABLE 4: Continued.

\begin{tabular}{|c|c|c|}
\hline Study & Measures of cognitive functions & Significant effect of target intervention group \\
\hline \multirow{12}{*}{ Takeuchi et al. [38] } & RAPM & 0 \\
\hline & Cattell culture fair test & 0 \\
\hline & DS & 0 \\
\hline & SpS & 0 \\
\hline & Tanaka B-type intelligence test & + \\
\hline & ST & 0 \\
\hline & rST & 0 \\
\hline & Simple arithmetic & 0 \\
\hline & Complex arithmetic & + \\
\hline & S-A creativity test & 0 \\
\hline & Word-color task & + \\
\hline & Color-word task & + \\
\hline \multirow{11}{*}{ Takeuchi et al. [62] } & RAPM & 0 \\
\hline & Arithmetic & 0 \\
\hline & Cd & 0 \\
\hline & rST & + \\
\hline & ST & 0 \\
\hline & S-A creativity test & - \\
\hline & Simple arithmetic & 0 \\
\hline & Complex arithmetic & + \\
\hline & Letter mental rotation & 0 \\
\hline & Trail making test B-A & 0 \\
\hline & Letter span & + \\
\hline \multirow{11}{*}{ Takeuchi et al. [63] } & RAPM & + \\
\hline & BOMAT & 0 \\
\hline & DS & + \\
\hline & SpS & + \\
\hline & Tanaka B-type intelligence test & + \\
\hline & Reverse Stroop interference & 0 \\
\hline & ST interference & + \\
\hline & Simple arithmetic & 0 \\
\hline & Complex arithmetic & 0 \\
\hline & Kyodai SX test & 0 \\
\hline & S-A creativity test & 0 \\
\hline \multirow{13}{*}{ Takeuchi et al. [64] } & RAPM & 0 \\
\hline & BOMAT & 0 \\
\hline & DS & 0 \\
\hline & SpS & 0 \\
\hline & Tanaka B-type intelligence test & 0 \\
\hline & Word-color Task & + \\
\hline & Color-word task & 0 \\
\hline & rST & 0 \\
\hline & ST & + \\
\hline & Simple arithmetic & 0 \\
\hline & Complex arithmetic & 0 \\
\hline & Kyodai SX test & 0 \\
\hline & S-A creativity test & + \\
\hline
\end{tabular}

MMSE: minimental state examination; RAPMT: Raven's advanced progressive matrices test; BOMAT: Bochumer Matrizen test; DAM (Goodenough draw-aman intelligence test); FAB: frontal assessment battery; WCST: Wisconsin card sorting test; ST: Stroop test; rST: reverse Stroop test; VFT: verbal fluency task; LM: logical memory; FSN: first and second names; DS: digit span; OpS: operation span; LNS: letter-number sequence; Ari: arithmetic; SpS: spatial span; DCAT: digit cancellation task; SRT: simple reaction task; Cd: symbol coding; SS: symbol search; JART: Japanese reading test; MR: mental rotation.

0 : no significant difference was found between the target intervention and control (intervention) groups; + : the target intervention group improved cognitive functions compared to the control (intervention) group; -: the control (intervention) group improved cognitive functions compared to the target intervention group.

Takeuchi et al. [38], who used processing speed training, published their findings in Journal of Neuroscience. Takeuchi et al. [62], who used working memory training with mental calculation, published their findings in PLoS One. 
computer buttons and difficulties (stimulus presentation rates) were modulated based on the subject performance. In some training tasks using computer buttons, a certain type of stimulus was presented successively. In each trial, subjects had to push buttons that corresponded with the presented stimuli before the next trial (before the next stimuli were presented). Stimuli were presented randomly as visual numbers (one, two, three, and four), visual locations (a mark was presented in one of four corners of the interface), auditory numbers (one, two, three, and four), and auditory locations (a pure tone was presented in either the left ear or the right ear). In one paper and pencil task, rows of pairs of number strings containing three single-digit numbers (zero to nine) were printed on paper. Participants had to decide whether the strings were the same when reordered. Participants were instructed to answer as many of these questions as possible in $1 \mathrm{~min}$. After a 6-day intervention period, the processing speed training group improved general cognitive ability/IQ measured by Tanaka B-types intelligence test, complex arithmetic, and processing speed measured by Word-Color and Color-Word tasks compared to the waiting list control group.

One working memory training study used mental calculations [62]. The study examined subjects in three groups: an intensive adaptive working memory training using mental calculation (adaptive working memory group), a nonadaptive working memory training using mental calculation (placebo group), and a no training group. In the adaptive working memory group, there were mental multiplication and mental addition tasks. For mental multiplication, participants were asked to solve mental multiplication problems in a normal way as participants do computations on paper in their minds and not to solve problems in any other way. Participants must continue the task until they get the correct answer. If participants answer correctly, then the problems become more difficult (the task starts from two-digit times two-digit multiplication and then becomes two-digit times three-digit multiplication and then three-digit times threedigit multiplication and then three-digit times four-digit mental multiplication). For mental addition task, ten twodigit numbers are presented one by one and the subjects are asked to add them. If they get the correct answer, the interstimulus interval (ISI) becomes shorter. In the placebo group, participants performed the similar tasks, except that the difficulty of the tasks does not change from the initial points (two-digit times two-digit multiplication in the mental multiplication task, ten-second ISI in the mental addition task). In the no training group, participants did not perform any training tasks. Results showed that the adaptive working memory training improved executive functions measured by Stroop test, working memory measured by letter span, and complex arithmetic. The adaptive working memory training decreased creativity measured by SA creativity test.

Another study used traditional working memory training tasks [63]. There were four working memory tasks: one visuospatial working memory, two types of dual working memory task, and one auditory backward operation span task. In all training tasks, the difficulties (number of items to be remembered) were modulated based on subjects' performance. For example, in the visuospatial working memory task, circles were presented one at a time at a $1 / \mathrm{s}$ rate in an interface where 10 squares were distributed irregularly (circles are presented in one of these squares). After stimuli presentation, the subjects indicated the location and order of the presented stimuli by clicking on a computer screen with a mouse. After 4 weeks of working memory training, general cognitive functions/IQ was measured by Raven's advanced progressive matrices test and Tanaka B-type intelligence test, working memory was measured by digit span and spatial span, and executive functions were measured by the Stroop test.

One study used multitask training [64]. Multitasking constituted simultaneous engagement in two or more cognitive activities. Six multitask training tasks existed. In all of these tasks, a certain type of stimulus was presented successively and randomly. In each trial, the subjects had to push multiple buttons on a keyboard that corresponded to the stimuli presented before the next trial (the next stimulus) was initiated. In all six training tasks, difficulties (stimulus presentation rates) were modulated based on the subjects' performance. For example, an auditory-visual dual task in which in each trial, one auditory stimulus $(1,2,3$, or 4$)$ is presented in English to both ears as well as one visual stimulus (a mark in one of four locations in a vertical row). The subjects must push "S" on the keyboard when they register stimulus 1 , “ $D$ " for 2 , " $F$ " for 3 , and " $G$ " for 4 . For the visual stimuli, the subjects must push " $H$ " for the leftmost stimulus, " $J$ " for the stimulus immediately after the first, and then " $K$ " and " $L$ " for the last two stimuli. Results showed that multiple training improved processing speed as measured by wordcolor task, executive functions measured by the Stroop test, and creativity measured by the S-A creativity test.

3.5.3. Exercise Training. One study examined combination exercise training for older people [65]. In the study, the combination exercise combined training of three types: aerobic, strength, and stretching. Participants performed the combination exercise training 3 days per week throughout the 4 weeks ( 12 workouts total). For the strength-training parts, participants were informed of the proper use of all of the equipment. They were instructed to complete as many repetitions as possible in a $30 \mathrm{~s}$ time period. There were twelve machines to exercise the whole body parts (chest press/seated row, squat, shoulder press/lat pull, leg extension/leg curl, abdominal crunch/back extension, lateral lift, elbow flexion/extension, horizontal leg press, pectoral deck, oblique, hip abductor/adductor, and gluteus). In a continuous interval fashion, participants performed floor-based aerobic training (e.g., running/skipping in place and arm circles) on recovery pads for a $30 \mathrm{~s}$ time period after each resistance exercise in an effort to maintain a consistent exercise heart rate corresponding to $60-80 \%$ of their heart maximum heart rate. Finally, participants did standardized whole-body stretching training (6 min). Whole-body stretching training consists of 12 stretching exercises (Achilles' tendon, sole of the foot, thigh, armpit, shoulder, shoulder/upper arm, chest/arm, shoulder/chest/arm, waist, back of knee, base of 
thigh, and back). Results of the combination exercise training for the older people demonstrated that the combination exercise training improved executive functions measured by verbal fluency task and the Stroop test, episodic memory measured by logical memory, and processing speed measured by symbol coding and symbol search compared to a waiting list control group.

3.5.4. Cognitive Training Using Paper-Pencil Programs. There were two studies using cognitive training with paper-pencil programs. One study was a reading aloud and simple calculation intervention program for older people [66]; the other study was a play intervention program for children [67]. Intervention programs aimed to improve functions of prefrontal cortex functions and created previous neuroimaging findings. Both studies used long-term intervention periods (3 or 6 months) and a waiting list control group.

One study for elderly people used simple cognitive intervention programs (learning therapy), which involved solving arithmetic and Japanese language problems [66]. These problems were used in everyday classes of first-grade to fourth-grade elementary school students. The problems were printed on both sides of an A4-size paper $(210 \times 297 \mathrm{~mm})$. As for the arithmetic problems, the lowest level of difficulty was single-digit addition, and the highest level was threedigit division. As for the Japanese language problems, the lowest level of difficulty was reading and writing simple sentences, and the highest level was reading fairy tales aloud. The participants were asked to go to classes once a week. They were then instructed to complete five sheets of each task prepared for each for that day, which were then assessed by the staff. The subjects were also asked to do their homework of two tasks for 4-6 days a week. As for their homework, the subjects were asked to complete five sheets of each task prepared for each individual. The results obtained using a paired $t$-test showed that learning therapy improved executive functions measured by frontal assessment battery and processing speed measured by symbol coding, but not the waiting list control group.

One study for the children used a play intervention program as cognitive training [67]. The play intervention programs consisted of a set of play activities based on standard cognitive tasks used in previous neuroimaging studies. The contents of the play activities were updated monthly (four play activities per month). The play intervention programs included the following five components. (1) For joint problem solving, the mothers and children developed mutual objectives through the play activities in our program. (2) The mothers and children were asked to alternate roles in the play activities to foster intersubjectivity. (3) To encourage warmth and responsiveness, the mothers were asked to be pleasant, warm, and responsive during the play activities. (4) To keep the children in the zone of proximal development (ZPD), which is the distance between the actual developmental level as determined by independent problem solving and the level of potential development as determined through problem solving under adult guidance or in collaboration with more capable peers, the difficulty levels of the play activities can be adjusted according to the children's ability levels. (5) To promote self-regulation, we asked mothers to be responsive to the children's contributions and to build on them according to children's initiative in the play activities. The results showed that children in the play intervention programs improved general cognitive function/IQ measured by the Goodenough draw-a-man intelligence test, working memory measured by the new S-S intelligence test, and processing speed measured by the new S-S intelligence test compared to the waiting list control group.

\section{Discussion}

In this systematic review, we evaluated the effectiveness of the intervention program on cognitive functions in healthy people. The review showed that various intervention programs conducted by our laboratory can improve cognitive functions such as executive functions, working memory, episodic memory, processing speed, and general cognitive ability/IQ.

A critical comparison between different intervention studies is difficult because of the heterogeneity of the intervention programs, participants, and outcome measures. However, results of this review suggest the following important things. First, cognitive functions can be improved by some intervention programs at any age. In this review, we identified improvements of cognitive functions in children [67], young people [38, 47, 63, 64], and older people [45, 65]. Although several studies investigated younger and older people (5 studies for young people and 3 studies for older people), only one study examined children. To elucidate important issues in this field, more research is needed using children. Second, cognitive functions can be changed rapidly through interventions. Two studies for young people demonstrated improvements of cognitive functions after 6 days of intervention $[38,62]$. However, it is important to note that the training time per day of these studies was quite long ( $4 \mathrm{hr}$ ) and that the total training time was $20 \mathrm{hr}$ through intervention periods. No short-term intervention studies (e.g., 6 days) have been made of children and older people. Additional studies must be undertaken to elucidate the mechanisms of this issue. Third, a systematic review showed that near transfer effects can often occur but not far transfer effects (see Table 4). For instance, brain training game intervention studies [45, 47], which require participants to use executive functions and processing speed, improved executive functions and processing speed but did not improve short-term memory. However, some studies have demonstrated near and far transfer effects. Two studies for the young people $[38,63]$ showed improvements of general cognitive function/IQ. Two studies for young people $[62,64]$ showed changes of creativity after cognitive intervention. The results indicated that the far transfer effects can occur after some cognitive training intervention. It is difficult to find and conclude the common characteristics of the far transfer effects among studies because of the heterogeneity of the intervention programs. It is expected to be necessary to find a critical component of the far transfer effects after interventions. 
For methodological quality, the average of the score of quality assessment was $9.89(\mathrm{SD}=1.27, \mathrm{Max}=14)$. That score denotes that the methodological quality of included studies was high. However, only four studies reported the CONSORT state $[45,47,65,67]$. The CONSORT statement is an evidence-based, minimum set of recommendations for reporting RCTs. The CONSORT statement includes a 25item checklist, which specifically examines reporting of how the trial was designed, analyzed, and interpreted. Therefore, future studies should use and report the CONSORT statement. Use of CONSORT might improve methodological quality and facilitate a meta-analysis study.

Recent study has recommended the use of an active control group [83]. Using the active control group can control or reduce effects of social interactions and new experiences of joining the intervention programs. A recent study demonstrated that waiting list control conditions may overestimate beneficial effects of interventions [84]. Given these facts, we must use the active control group instead of a no-intervention group or a waiting-list control group. When examining the studies included studies in the review, three studies used the active control group [45, 47, 62]. These studies clearly revealed improvements of cognitive functions compared to the active control group. Based on those results, it may be concluded that intervention programs using a brain training game and working memory training can have positive effects on cognitive functions. It remains unclear how beneficial effects of interventions on cognitive functions in children compared to an active control group because of few studies have used an active control group. In the future, additional studies using an active control group for children are needed.

It is important to consider sample size in intervention programs. For the clinical trial, some phases are used to test the effectiveness and evidence of a new intervention program. Clinical trials involving new intervention programs are commonly classified into four phases (Phases 1-4) [85]. In Phase 1 trials, researchers test an intervention program in a very small group of people $(20-80)$ to evaluate its safety, to determine an intervention a period, and to identify side effects. In Phase 2 trials, the experimental treatment is administered to a somewhat larger group of people (100-300) to elucidate whether it is effective compared with a placebo or a no-intervention control group or not and to further evaluate its safety. In Phase 3 trials, the treatment is given to large groups of people $(1,000-3,000)$ to confirm its effectiveness, to monitor side effects, to compare it to other effective intervention programs, and to collect information that will allow it to be used safely. In Phase 4 trials, postmarketing studies delineate additional information, including the treatment's risks, benefits, and optimal use. Studies of intervention programs for improvements of cognitive functions may not fit into a single phase. For example, some may blend from Phase 1 to Phase 2 or from Phase 2 to Phase 3. When looking at the included studies in the review, the purpose of some studies met the study of Phase 2 or the blend study of Phases 2 and 3. However, several studies examined a small sample (fewer than 60). The sample sizes differed among the included studies (from 32 to 238 , average $=76.67, \mathrm{SD}=66.66$ ).
To generalize the effectiveness of the intervention program (Phases 3 or 4 ), we should conduct a large sample study.

An important limitation is the following. We did not conduct a meta-analysis of the effect size among the included studies because of the heterogeneity of intervention programs and cognitive measures. For future research, inclusion of a core set of outcome measures would be necessary to compare the effectiveness of different cognitive intervention programs. Outcome measures for basic cognitive functions such as subscales of WAIS should be part of this core set of outcome measures.

This systematic review showed that unresolved issues exist. First, the issue of whether the effects of some interventions programs generalize to improvement in everyday life activities such as driving skill is still unresolved and needs to be addressed more explicitly in future research. Second, it remains unclear whether intervention programs can improve cognitive functions in people who have any diagnosis such as developmental disorder, depression, posttraumatic stress disorder (PTSD), dementia, mild cognitive impairment, or Alzheimer's disease. Our previous studies showed that cognitive training using reading aloud improved cognitive functions in older people with dementia [78] and children with autism spectrum disorder [79]. To confirm the issue, we should conduct the intervention study with nonhealthy people as well as healthy people.

\section{Conclusion}

Cognitive functions are important for our daily life in any age. Consequently, we started the Smart Ageing research, which investigates how to improve cognitive functions. The conclusion of this systematic review was summarized as follows. (1) The intervention programs for Smart Ageing can be effective for improving various aspects of cognitive function (executive functions, working memory, episodic memory, processing speed, and general cognitive ability) in the healthy children, young people, and older people. (2) The cognitive functions can be changed rapidly by the short-term intervention such as 6 days. (3) The near transfer effects can often occur but not the far transfer effects. (4) The methodological quality of the included studies was high. The systematic review also indicated the following limitations and unresolved issues. (1) We did not conduct a meta-analysis for the effect size due to the heterogeneity of methods and outcome measures. (2) It is unresolved whether our intervention programs have beneficial effects on the everyday activities such as driving skill. (3) It remains unclear whether our intervention programs could improve cognitive function in the nonhealthy populations such as children with developmental disorders and older people with dementia. To overcome these problems, Smart Ageing research must be continued.

\section{Conflict of Interests}

The authors declare that there is no conflict of interests regarding the publication of this paper. 


\section{Acknowledgments}

The authors thank Haruka Nouchi for searching for papers and all their other colleagues at IDAC, Tohoku University, for their support. This study was supported by JST/RISTEX and JST/CREST.

\section{References}

[1] T. Hedden and J. D. E. Gabrieli, "Insights into the ageing mind: a view from cognitive neuroscience," Nature Reviews Neuroscience, vol. 5, no. 2, pp. 87-96, 2004.

[2] S. Mejia, D. Pineda, L. M. Alvarez, and A. Ardila, "Individual differences in memory and executive function abilities during normal aging," International Journal of Neuroscience, vol. 95, no. 3-4, pp. 271-284, 1998.

[3] L. G. Nilsson, "Memory function in normal aging," Acta Neurologica Scandinavica, Supplement, vol. 107, supplement 179, pp. 7-13, 2003.

[4] R. C. Petersen, G. Smith, E. Kokmen, R. J. Ivnik, and E. G. Tangalos, "Memory function in normal aging," Neurology, vol. 42, no. 2, pp. 396-401, 1992.

[5] S. Blakemore and S. Choudhury, "Development of the adolescent brain: Implications for executive function and social cognition," Journal of Child Psychology and Psychiatry and Allied Disciplines, vol. 47, no. 3-4, pp. 296-312, 2006.

[6] D. C. Park, G. Lautenschlager, T. Hedden, N. S. Davidson, A. D. Smith, and P. K. Smith, "Models of visuospatial and verbal memory across the adult life span," Psychology and Aging, vol. 17, no. 2, pp. 299-320, 2002.

[7] T. A. Salthouse, "Memory aging from 18 to 80," Alzheimer Disease and Associated Disorders, vol. 17, no. 3, pp. 162-167, 2003.

[8] N. N. Yakhno, V. V. Zakharov, and A. B. Lokshina, "Impairment of memory and attention in the elderly," Neuroscience and Behavioral Physiology, vol. 37, no. 3, pp. 203-208, 2007.

[9] A. K. Coppin, A. Shumway-Cook, J. S. Saczynski et al., "Association of executive function and performance of dual-task physical tests among older adults: analyses from the InChianti study," Age and Ageing, vol. 35, no. 6, pp. 619-624, 2006.

[10] D. R. Royall, R. Palmer, L. K. Chiodo, and M. J. Polk, "Declining executive control in normal aging predicts change in functional status: the freedom house study," Journal of the American Geriatrics Society, vol. 52, no. 3, pp. 346-352, 2004.

[11] T. A. Salthouse, "The processing-speed theory of adult age differences in cognition," Psychological Review, vol. 103, no. 3, pp. 403-428, 1996.

[12] H. L. Swanson and M. Beebe-Frankenberger, "The relationship between working memory and mathematical problem solving in children at risk and not at risk for serious math difficulties," Journal of Educational Psychology, vol. 96, no. 3, pp. 471-491, 2004.

[13] H. L. Swanson and C. Sachse-Lee, "Mathematical problem solving and working memory in children with learning disabilities: both executive and phonological processes are important," Journal of Experimental Child Psychology, vol. 79, no. 3, pp. 294321, 2001.

[14] T. E. Rohde and L. A. Thompson, "Predicting academic achievement with cognitive ability," Intelligence, vol. 35, no. 1, pp. 83-92, 2007.

[15] W. S. Barnett, "Long-term cognitive and academic effects of early childhood education on children in poverty," Preventive Medicine, vol. 27, no. 2, pp. 204-207, 1998.
[16] F. A. Campbell, E. P. Pungello, S. Miller-Johnson, M. Burchinal, and C. T. Ramey, "The development of cognitive and academic abilities: growth curves from an early childhood educational experiment," Developmental Psychology, vol. 37, no. 2, pp. 231242, 2001.

[17] H. W. Stevenson, J. W. Stigler, S. Y. Lee, G. W. Lucker, S. Kitamura, and C. C. Hsu, "Cognitive performance and academic achievement of Japanese, Chinese, and American children," Child Development, vol. 56, no. 3, pp. 718-734, 1985.

[18] P. Barberger-Gateau and C. Fabrigoule, "Disability and cognitive impairment in the elderly," Disability and Rehabilitation, vol. 19, no. 5, pp. 175-193, 1997.

[19] D. A. Cahn-Weiner, P. F. Malloy, P. A. Boyle, M. Marran, and S. Salloway, "Prediction of functional status from neuropsychological tests in community-dwelling elderly individuals," Clinical Neuropsychologist, vol. 14, no. 2, pp. 187-195, 2000.

[20] M. C. Carlson, L. P. Fried, Q. Xue, K. Bandeen-Roche, S. L. Zeger, and J. Brandt, "Association between executive attention and physical functional performance in community-dwelling older women," Journals of Gerontology B: Psychological Sciences and Social Sciences, vol. 54, no. 5, pp. S262-S270, 1999.

[21] J. Grigsby, K. Kaye, J. Baxter, S. M. Shetterly, and R. F. Hamman, "Executive cognitive abilities and functional status among community-dwelling older persons in the San Luis Valley health and aging study," Journal of the American Geriatrics Society, vol. 46, no. 5, pp. 590-596, 1998.

[22] Y. Lee, J. H. Kim, K. J. Lee, G. Han, and J. L. Kim, “Association of cognitive status with functional limitation and disability in older adults," Aging Clinical and Experimental Research, vol. 17, no. 1, pp. 20-28, 2005.

[23] C. Owsley and G. McGwin Jr., "Association between visual attention and mobility in older adults," Journal of the American Geriatrics Society, vol. 52, no. 11, pp. 1901-1906, 2004.

[24] P. Verhaeghen, A. Marcoen, and L. Goossens, "Improving memory performance in the aged through mnemonic training: a meta-analytic study," Psychology and Aging, vol. 7, no. 2, pp. 242-251, 1992.

[25] S. Colcombe and A. F. Kramer, "Fitness effects on the cognitive function of older adults: a meta-analytic study," Psychological Science, vol. 14, no. 2, pp. 125-130, 2003.

[26] C. Lustig, P. Shah, R. Seidler, and P. A. Reuter-Lorenz, "Aging, training, and the brain: a review and future directions," Neuropsychology Review, vol. 19, no. 4, pp. 504-522, 2009.

[27] C. S. Green and D. Bavelier, "Exercising your brain: a review of human brain plasticity and training-induced learning," Psychology and Aging, vol. 23, no. 4, pp. 692-701, 2008.

[28] H. Takeuchi, Y. Taki, and R. Kawashima, "Effects of working memory training on cognitive functions and neural systems," Reviews in the Neurosciences, vol. 21, no. 6, pp. 427-449, 2010.

[29] M. Valenzuela and P. Sachdev, "Can cognitive exercise prevent the onset of dementia? Systematic review of randomized clinical trials with longitudinal follow-up," The American Journal of Geriatric Psychiatry, vol. 17, no. 3, pp. 179-187, 2009.

[30] K. V. Papp, S. J. Walsh, and P. J. Snyder, "Immediate and delayed effects of cognitive interventions in healthy elderly: a review of current literature and future directions," Alzheimer's and Dementia, vol. 5, no. 1, pp. 50-60, 2009.

[31] L. Jean, M. Bergeron, S. Thivierge, and M. Simard, "Cognitive intervention programs for individuals with mild cognitive impairment: systematic review of the literature," The American Journal of Geriatric Psychiatry, vol. 18, no. 4, pp. 281-296, 2010. 
[32] P. J. Smith, J. A. Blumenthal, B. M. Hoffman et al., "Aerobic exercise and neurocognitive performance: a meta-analytic review of randomized controlled trials," Psychosomatic Medicine, vol. 72, no. 3, pp. 239-252, 2010.

[33] C.-N. Tseng, B.-S. Gau, and M.-F. Lou, "The effectiveness of exercise on improving cognitive function in older people: a systematic review," Journal of Nursing Research, vol. 19, no. 2, pp. 119-131, 2011.

[34] Y.-K. Chang, C.-Y. Pan, F.-T. Chen, C.-L. Tsai, and C.-C. Huang, "Effect of resistance-exercise training on cognitive function in healthy older adults: a review," Journal of Aging and Physical Activity, vol. 20, no. 4, pp. 497-517, 2012.

[35] M. N. McDonnell, A. E. Smith, and S. F. MacKintosh, "Aerobic exercise to improve cognitive function in adults with neurological disorders: a systematic review," Archives of Physical Medicine and Rehabilitation, vol. 92, no. 7, pp. 1044-1052, 2011.

[36] J. G. Z. van Uffelen, M. J. M. Chin A Paw, M. Hopman-Rock, and W. van Mechelen, "The effects of exercise on cognition in older adults with and without cognitive decline: a systematic review," Clinical Journal of Sport Medicine, vol. 18, no. 6, pp. 486-500, 2008.

[37] L. Clare and R. T. Woods, "Cognitive training and cognitive rehabilitation for people with early-stage Alzheimer's disease: a review," Neuropsychological Rehabilitation, vol. 14, no. 4, pp. 385-401, 2004.

[38] H. Takeuchi, Y. Taki, H. Hashizume et al., "Effects of training of processing speed on neural systems," The Journal of Neuroscience, vol. 31, no. 34, pp. 12139-12148, 2011.

[39] T. Klingberg, "Training and plasticity of working memory," Trends in Cognitive Sciences, vol. 14, no. 7, pp. 317-324, 2010.

[40] L. L. Richmond, A. B. Morrison, J. M. Chein, and I. R. Olson, "Working memory training and transfer in older adults," Psychology and Aging, vol. 26, no. 4, pp. 813-822, 2011.

[41] J. D. Edwards, V. G. Wadley, D. E. Vance, K. Wood, D. L. Roenker, and K. K. Ball, "The impact of speed of processing training on cognitive and everyday performance," Aging \& Mental Health, vol. 9, no. 3, pp. 262-271, 2005.

[42] B. Carretti, E. Borella, and R. De Beni, "Does strategic memory training improve the working memory performance of younger and older adults?" Experimental Psychology, vol. 54, no. 4, pp. 311-320, 2007.

[43] D. F. Bjorklund, P. H. Miller, T. R. Coyle, and J. L. Slawinski, "Instructing children to use memory strategies: Evidence of utilization deficiencies in memory training studies," Developmental Review, vol. 17, no. 4, pp. 411-441, 1997.

[44] H. W. Mahncke, B. B. Connor, J. Appelman et al., "Memory enhancement in healthy older adults using a brain plasticitybased training program: a randomized, controlled study," Proceedings of the National Academy of Sciences of the United States of America, vol. 103, no. 33, pp. 12523-12528, 2006.

[45] R. Nouchi, Y. Taki, H. Takeuchi et al., "Brain training game improves executive functions and processing speed in the elderly: a randomized controlled trial," PLoS ONE, vol. 7, no. 1, Article ID e29676, 2012.

[46] D. J. Miller and D. P. Robertson, "Using a games console in the primary classroom: Effects of 'Brain Training' programme on computation and self-esteem," British Journal of Educational Technology, vol. 41, no. 2, pp. 242-255, 2010.

[47] R. Nouchi, Y. Taki, H. Takeuchi et al., "Brain training game boosts executive functions, working memory and processing speed in the young adults: a randomized controlled trial," PLoS ONE, vol. 8, no. 2, Article ID e55518, 2013.
[48] S. Bergman Nutley, S. Söderqvist, S. Bryde, L. B. Thorell, K. Humphreys, and T. Klingberg, "Gains in fluid intelligence after training non-verbal reasoning in 4-year-old children: a controlled, randomized study," Developmental Science, vol. 14, no. 3, pp. 591-601, 2011.

[49] D. J. Miller and D. P. Robertson, "Educational benefits of using game consoles in a primary classroom: a randomised controlled trial," British Journal of Educational Technology, vol. 42, no. 5, pp. 850-864, 2011.

[50] J. L. Mozolic, A. B. Long, A. R. Morgan, M. Rawley-Payne, and P. J. Laurienti, "A cognitive training intervention improves modality-specific attention in a randomized controlled trial of healthy older adults," Neurobiology of Aging, vol. 32, no. 4, pp. 655-668, 2011.

[51] K. Ball, D. B. Berch, K. F. Helmers et al., "Effects of cognitive training interventions with older adults: a randomized controlled trial," Journal of the American Medical Association, vol. 288, no. 18, pp. 2271-2281, 2002.

[52] R. C. Cassilhas, V. A. R. Viana, V. Grassmann et al., "The impact of resistance exercise on the cognitive function of the elderly," Medicine \& Science in Sports and Exercise, vol. 39, no. 8, pp. 1401-1407, 2007.

[53] P. Perrig-Chiello, W. J. Perrig, R. Ehrsam, H. B. Staehelin, and F. Krings, "The effects of resistance training on well-being and memory in elderly volunteers," Age and Ageing, vol. 27, no. 4, pp. 469-475, 1998.

[54] C. L. Davis, P. D. Tomporowski, J. E. McDowell et al., "Exercise improves executive function and achievement and alters brain activation in overweight children: a randomized, controlled trial," Health Psychology, vol. 30, no. 1, pp. 91-98, 2011.

[55] J. D. Bransford, A. L. Brown, and R. R. Cocking, How People Learn, National Academy Press, Washington, DC, USA, 2000.

[56] S. M. Barnett and S. J. Ceci, "When and where do we apply what we learn? A taxonomy for far transfer," Psychological Bulletin, vol. 128, no. 4, pp. 612-637, 2002.

[57] E. M. Zelinski, "Far transfer in cognitive training of older adults," Restorative Neurology and Neuroscience, vol. 27, no. 5, pp. 455-471, 2009.

[58] J. D. Edwards, V. G. Wadley, R. S. Myers, D. L. Roenker, G. M. Cissell, and K. K. Ball, "Transfer of a speed of processing intervention to near and far cognitive functions," Gerontology, vol. 48, no. 5, pp. 329-340, 2002.

[59] J. Karbach and J. Kray, "How useful is executive control training? Age differences in near and far transfer of taskswitching training," Developmental Science, vol. 12, no. 6, pp. 978-990, 2009.

[60] A. Liberati, D. G. Altman, J. Tetzlaff et al., "The PRISMA statement for reporting systematic reviews and meta-analyses of studies that evaluate health care interventions: explanation and elaboration," Annals of Internal Medicine, vol. 151, no. 4, pp. 65-94, 2009.

[61] A. P. Verhagen, H. C. W. De Vet, R. A. De Bie et al., “The Delphi list: a criteria list for quality assessment of randomized clinical trials for conducting systematic reviews developed by Delphi consensus," Journal of Clinical Epidemiology, vol. 51, no. 12, pp. 1235-1241, 1998.

[62] H. Takeuchi, Y. Taki, Y. Sassa et al., "Working memory training using mental calculation impacts regional gray matter of the frontal and parietal regions," PLOS ONE, vol. 6, no. 8, Article ID e23175, 2011. 
[63] H. Takeuchi, Y. Taki, R. Nouchi et al., "Effects of working memory training on functional connectivity and cerebral blood flow during rest," Cortex, vol. 49, no. 8, pp. 2106-2125, 2013.

[64] H. Takeuchi, Y. Taki, R. Nouchi et al., "Anatomical correlates of quality of life: evidence from voxel-based morphometry," Human Brain Mapping, vol. 35, no. 5, pp. 1834-1846, 2014.

[65] R. Nouchi, Y. Taki, H. Takeuchi et al., "Four weeks of combination exercise training improved executive functions, episodic memory, and processing speed in healthy elderly people: evidence from a randomized controlled trial," AGE, vol. 36, no. 2, pp. 787-799, 2014.

[66] S. Uchida and R. Kawashima, "Reading and solving arithmetic problems improves cognitive functions of normal aged people: a randomized controlled study," AGE, vol. 30, no. 1, pp. 21-29, 2008.

[67] Y. Tachibana, A. Fukushima, H. Saito, S. Yoneyama, K. Ushida, and R. Kawashima, "A new mother-child play activity program to decrease parenting stress and improve child cognitive abilities: a cluster randomized controlled trial," PLoS ONE, vol. 7, no. 7, Article ID e38238, 2012.

[68] R. Kawashima, "Mental exercises for cognitive function: clinical evidence," Journal of Preventive Medicine and Public Health, vol. 46, supplement 1, pp. S22-S27, 2013.

[69] H. Takeuchi and R. Kawashima, "Effects of processing speed training on cognitive functions and neural systems," Reviews in the Neurosciences, vol. 23, no. 3, pp. 289-301, 2012.

[70] Y. Tachibana, Y. Akitsuki, and R. Kawashima, "Cognitive interventions to imporve prfrontal functions," Brain Research Journal, vol. 3, no. 3-4, pp. 185-205, 2011.

[71] R. Nouchi, Y. Taki, H. Takeuchi et al., "Beneficial effects of shortterm combination exercise training on diverse cognitive functions in healthy older people: study protocol for a randomized controlled trial," Trials, vol. 13, article 200, 2012.

[72] R. Nouchi, Y. Taki, H. Takeuchi et al., "Beneficial effects of reading aloud and solving simple arithmetic calculations (learning therapy) on a wide range of cognitive functions in the healthy elderly: study protocol for a randomized controlled trial," Trials, vol. 13, article 32, 2012.

[73] Y. Tachibana, J. Yoshida, M. Ichinomiya et al., "A GO intervention program for enhancing elementary school children's cognitive functions and control abilities of emotion and behavior: study protocol for a randomized controlled trial," Trials, vol. 13, article 8, 2012.

[74] N. Maionchi-Pino, Y. Taki, S. Yokoyama et al., "Is the phonological deficit in developmental dyslexia related to impaired phonological representations and to universal phonological grammar?" Journal of Experimental Child Psychology, vol. 115, no. 1, pp. 53-73, 2013.

[75] H. Takeuchi, M. Sugiura, Y. Sassa et al., "Neural correlates of the difference between working memory speed and simple sensorimotor speed: an fMRI study," PLoS ONE, vol. 7, no. 1, Article ID e30579, 2012.

[76] S. Kanoh, Y. M. Murayama, K. Miyamoto, T. Yoshinobu, and R. Kawashima, "A NIRS-based brain-computer interface system during motor imagery: system development and online feedback training," in Proceedings of the Annual International Conference of the IEEE Engineering in Medicine and Biology Society, vol. 2009, pp. 594-597, 2009.

[77] H. Takeuchi, A. Sekiguchi, Y. Taki et al., "Training of working memory impacts structural connectivity," Journal of Neuroscience, vol. 30, no. 9, pp. 3297-3303, 2010.
[78] R. Kawashima, K. Okita, R. Yamazaki et al., "Reading aloud and arithmetic calculation improve frontal function of people with dementia," Journals of Gerontology A: Biological Sciences and Medical Sciences, vol. 60, no. 3, pp. 380-384, 2005.

[79] Y. Tachibana, Y. Hwang, Y. Abe, S. Goto, K. Sugai, and R. Kawashima, "Reading aloud improves executive function of children with autism spectrum disorder: a pilot randomized controlled trial," International Journal on Disability and Human Development, vol. 12, no. 1, pp. 91-101, 2013.

[80] R. Kawashima, "A new intervention program for improvement of cognitive functions of senile dementia patients," Seishin Shinkeigaku Zasshi, vol. 107, no. 12, pp. 1305-1309, 2005.

[81] R. Kawashima, "Cognitive rehabilitation for Alzheimer disease-the learning therapy," Rinsho Shinkeigaku, vol. 45, no. 11, pp. 864-866, 2005.

[82] A. Sekiguchi and R. Kawashima, "Cognitive rehabilitation-the learning therapy for the senile dementia," Brain and Nerve, vol. 59, no. 4, pp. 357-365, 2007.

[83] Z. Shipstead, T. S. Redick, and R. W. Engle, "Is working memory training effective?" Psychological Bulletin, vol. 138, no. 4, pp. 628-654, 2012.

[84] J. A. Cunningham, K. Kypri, and J. McCambridge, "Exploratory randomized controlled trial evaluating the impact of a waiting list control design," BMC Medical Research Methodology, vol.13, no. 1, article 150, 2013.

[85] L. M. Friedman, C. Furberg, and D. L. DeMets, Fundamentals of Clinical Trials, Springer, New York, NY, USA, 1998. 

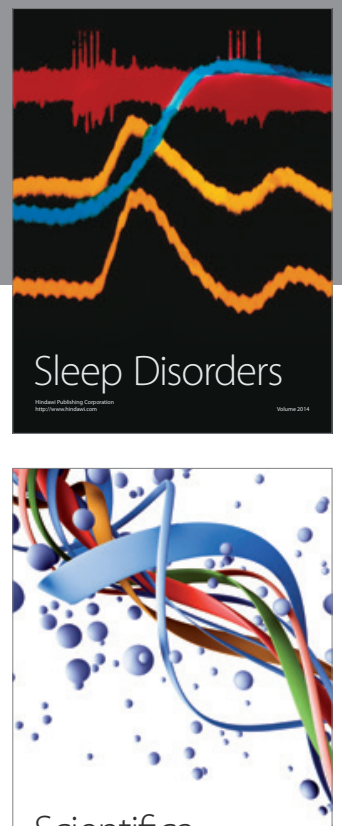

Scientifica
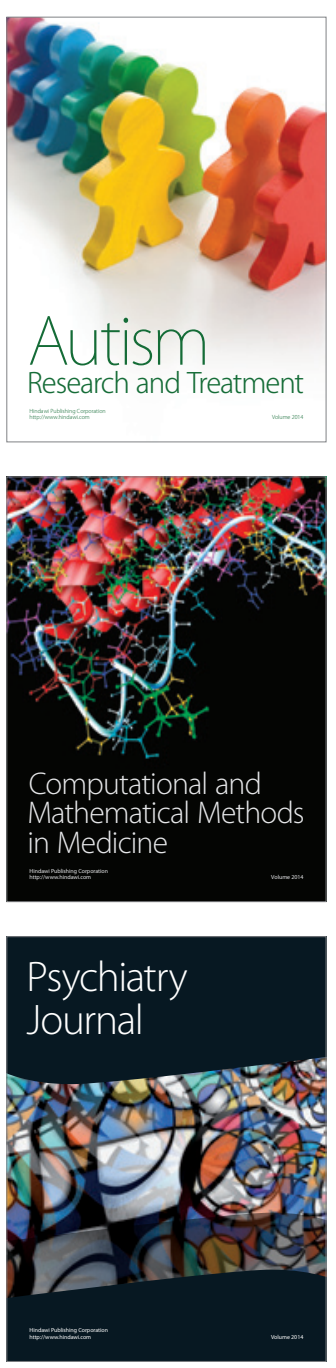
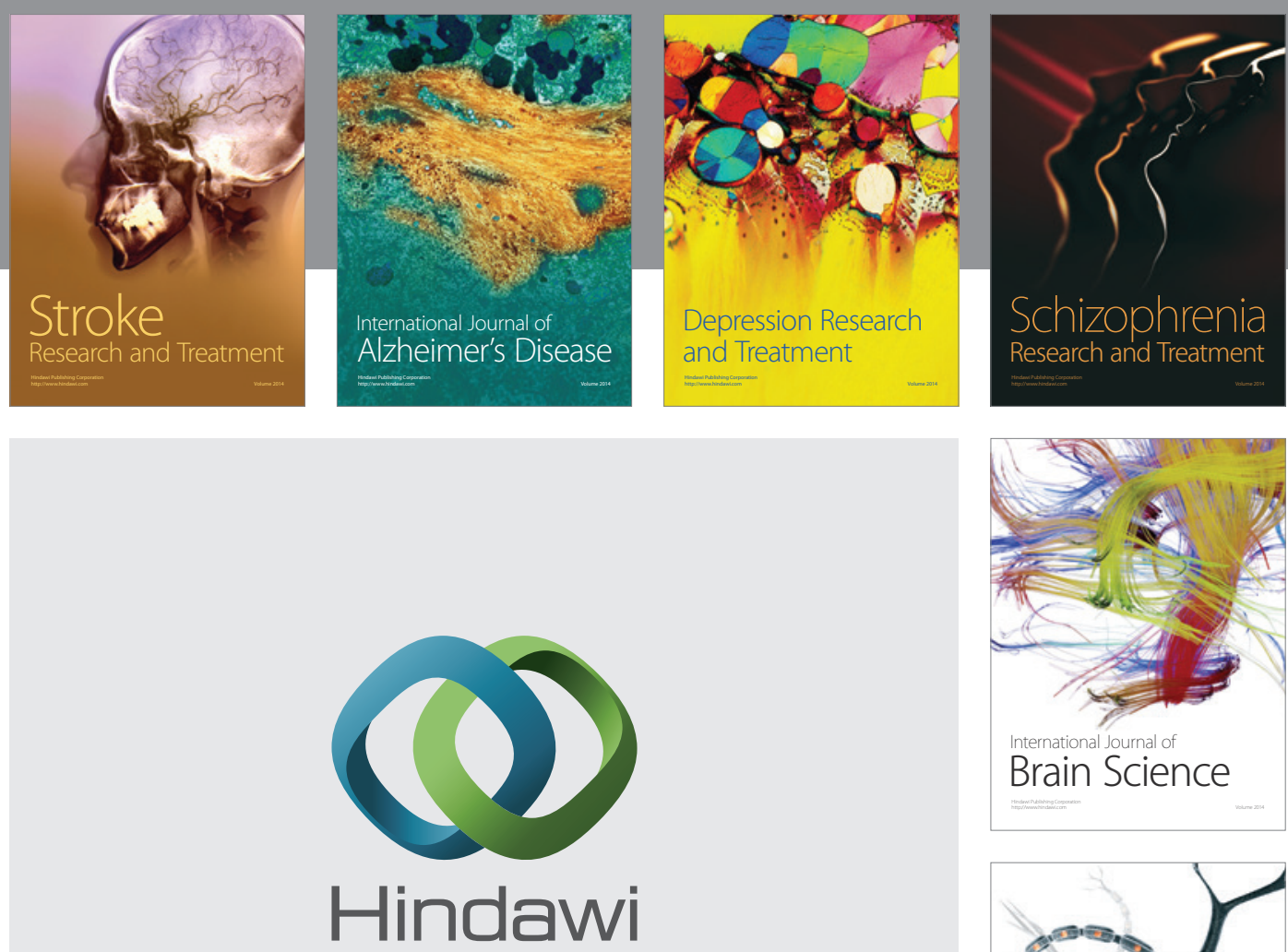

Submit your manuscripts at

http://www.hindawi.com
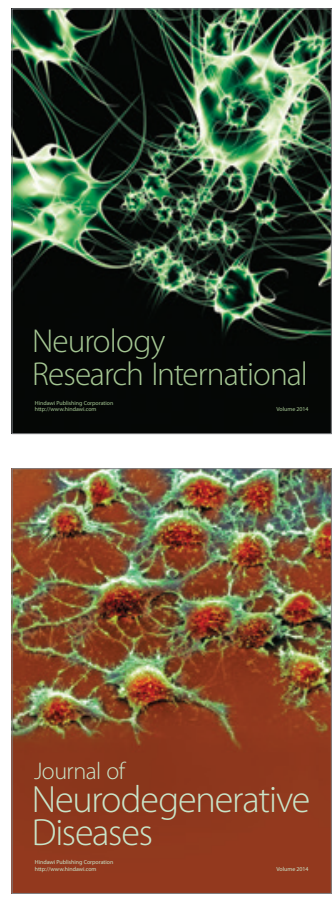

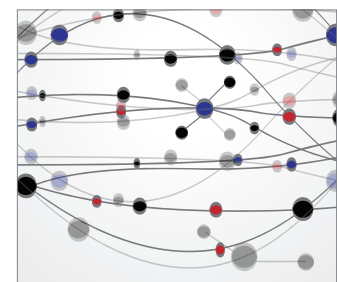

The Scientific World Journal
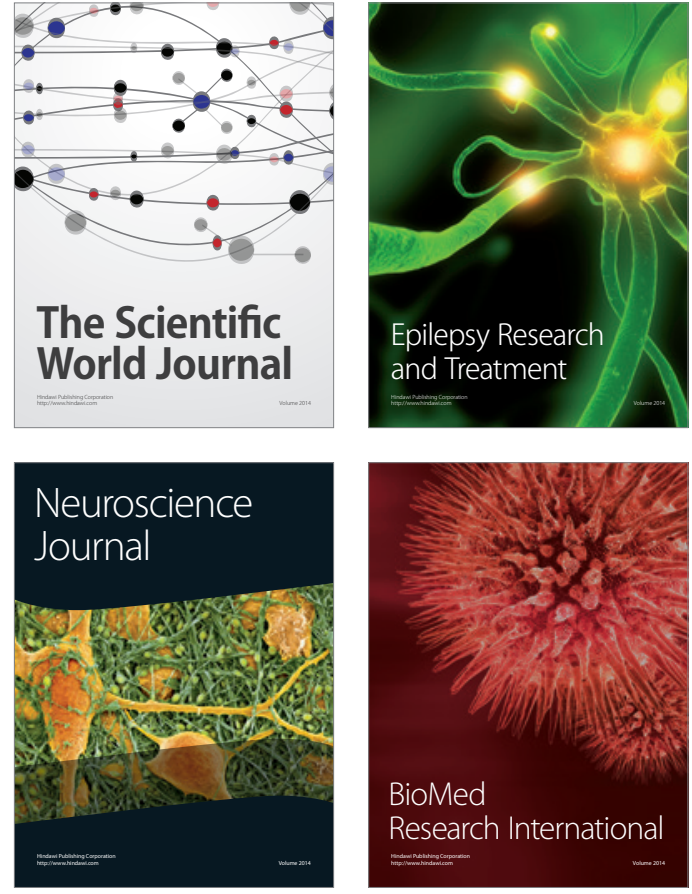

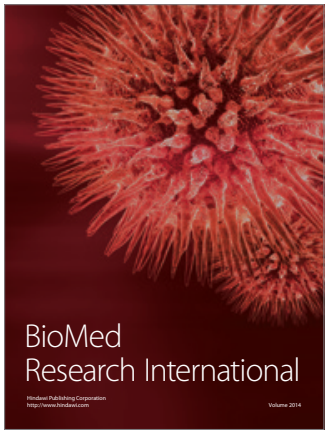

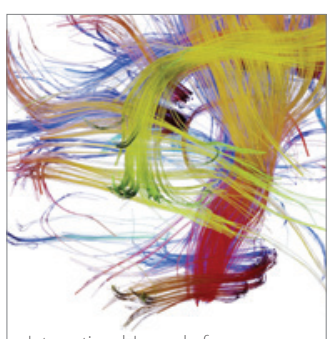

Brain Science

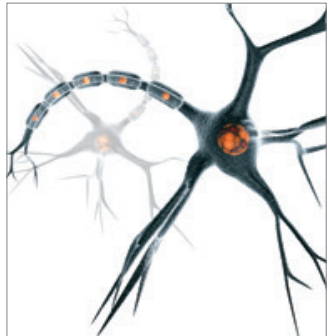

Neural Plasticity
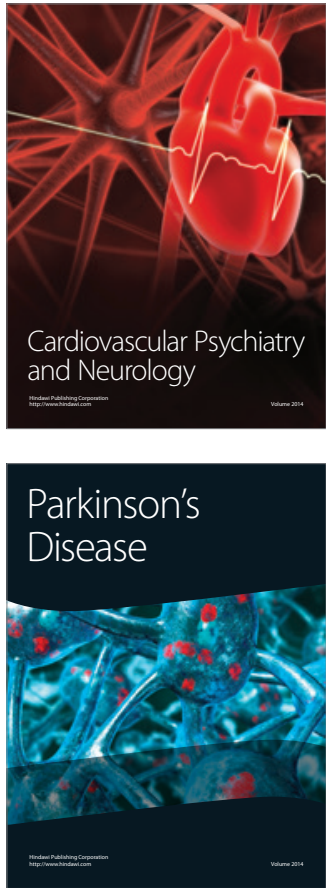